Vol. 43 (1991) [303-306]

\title{
OPEN IMAGES OF COMPACTIFICATIONS OF THE RAY
}

\author{
Marwan M. Awartani
}

Let $X$ be a compactification of the ray with the arc as remainder. The following characterisation of the open images of $X$ is obtained: Let $h: X \rightarrow Y$ be an open onto map. If $Y$ is not homeomorphic to $[0,1]$ or the one-point space, then $h$ is a homeomorphism. In 1977 open images of the usual $\sin (1 / x)$ continuum were characterised by Professor Sam B. Nadler.

\section{INTRODUCTION}

A continuum is a compact connected metric space. By a map we mean a continuous function. A map $f$ from a continuum $X$ onto a continuum $Y$ is said to be

(i) confluent provided that for each subcontinuum $L$ of $Y$ and each component $K$ of $f^{-1}(L)$, we have $f(K)=L$;

(ii) monotone provided that $f^{-1}(y)$ is connected for each $y \in Y$;

(iii) open provided that $f(A)$ is open in $Y$ for each open subset $A$ of $X$.

Note that monotone and open maps between continua are confluent [3].

Addressing the following question that arose in a conversation between Professor J.J. Charatonik and Professor Sam B. Nadler "What are all open images of the $\sin (1 / x)$ continuum (the closure of the graph of the function $\sin (1 / x), 0<x \leqslant 1$ )?", Professor Nadler obtained the following (stronger) result:

THEOREM 1.1. [6] If $Y$ is a confluent image of $S$ (the $\sin (1 / x)$ continuum), then $Y$ is homeomorphic to $[0,1], S$, or a one-point space (and conversely).

Nadler's proof depends upon distinguishing the $\sin (1 / x)$ continuum as the only compactification of the ray with the arc as remainder which has property $[\kappa]$, and then using the fact that property $[\kappa]$ is a confluent invariant. Property $[\kappa]$ was first introduced by Kelley [4] and was shown to be a confluent invariant by Wardle [9]. In this paper, the following generalisation of the above theorem is obtained:

THEOREM 1.2. Let $X$ be a compactification of the ray with the arc as remainder, and let $h: X \rightarrow Y$ be an open onto map. If $Y$ is not homeomorphic to $[0,1]$ or the one-point space, then $h$ is a homeomorphism.

Notice that the class of compactifications of the ray with the arc as remainder is quite rich and its members can have fairly complex topological structure [1]. In fact it

Received 1 May 1990

Copyright Clearance Centre, Inc. Serial-fee code: 0004-9729/91 $\$ \mathbf{A 2 . 0 0 + 0 . 0 0}$. 
is established in [2] that there exist continuum many compactifications of the ray with the arc as remainder no one of which maps onto any other. The $\sin (1 / x)$ continuum is obviously the simplest such compactification.

It is also shown that Theorem 1.1 does not hold for an arbitrary compactification of the ray with the arc as remainder and Theorem 1.2 does not hold for confluent (or even monotone) maps.

\section{The ReSUlts}

THEOREM 2.1. Let $X$ be a compactification of the ray with the arc as remainder and let $h: X \rightarrow Y$ be an open onto map. If $Y$ is not homeomorphic to $[0,1]$ or the one-point space, then $Y$ is a compactification of the ray with the arc as remainder.

Proof: Since $X$ is hereditarily decomposable and chainable, it follows from [5, p.94] that $Y$ is chainable. If $Y$ is pathwise connected and nondegenerate, then $Y$ is homeomorphic to $[0,1],[7, \mathrm{p} .230]$. If $Y$ is not pathwise connected, then it must consist of exactly two path components. By Theorem 1 of $[8, p .188]$, one of the components is an arc and the other is a ray, say $J_{Y}$. Let $J_{X}$ denote the ray densely embedded in $X$.

Since $h$ is an onto map, $h\left(X-J_{X}\right)=J_{Y}$ or $Y-J_{Y}$. But since $X-J_{X}$ is compact and $J_{Y}$ is not, $h\left(X-J_{X}\right)=Y-J_{Y}$ and hence $h\left(J_{X}\right)=J_{Y}$. Then $Y=h \overline{\left(J_{X}\right)} \subseteq$ $\overline{h\left(J_{X}\right)}=J_{Y}$. Hence $J_{Y}$ is dense in $Y$, implying that $Y$ is a compactification of the ray $J_{Y}$ with the arc as remainder.

LEMmA 2.2. Let $X$ and $Y$ be compactifications of the ray with the arc as remainder. If $h: X \rightarrow Y$ is an open onto map, then $h$ is one-to-one.

Proof: The proof is broken into two steps:

STEP 1. First it is shown that $h \mid J_{X}: J_{X} \rightarrow J_{Y}$ is one-to-one, where $J_{X}$ and $J_{Y}$ denote the rays densely embedded in $X$ and $Y$ respectively. As indicated in the proof of Theorem 2.1, $h\left(J_{X}\right)=J_{Y}$ and $h\left(X-J_{X}\right)=Y-J_{Y}$. Suppose that $h \mid J_{X}$ is not one-to-one, and let $y \in J_{Y}$ such that $h^{-1}(y)$ is not a singleton. Since $h$ is continuous, $h^{-1}(y)$ is closed, and since $h$ is open, $h^{-1}(y)$ is nowhere dense in $J_{X}$, Hence points $t_{1}$ and $t_{2}$ can be chosen in $h^{-1}(y)$ such that $\left(t_{1}, t_{2}\right) \cap h^{-1}(y)=\emptyset$. Then $h\left[t_{1}, t_{2}\right]$ is a closed arc in $J_{Y}$ having $y$ as one endpoint. Let $y^{\prime}$ denote the other endpoint of $h\left[t_{1}, t_{2}\right]$. Then $h\left(t_{1}, t_{2}\right)$ is an arc in $J_{Y}$ having finite length and having exactly one endpoint, namely $y^{\prime}$, implying that $h$ is not open. This is a contradiction.

Step 2. $h \mid X-J_{X}$ is one-to-one. Suppose that there exist points $x_{1}$ and $x_{2}$ in $X-J_{X}$ such that $h\left(x_{1}\right)=h\left(x_{2}\right)=y$. Let $G_{1}$ and $G_{2}$ be disjoint open neighbourhoods of $x_{1}$ and $x_{2}$ respectively. Then $h\left(G_{1}\right) \cap h\left(G_{2}\right)$ is an open neighbourhood of $y$. Hence $h\left(G_{1}\right) \cap h\left(G_{2}\right) \cap J_{Y} \neq \emptyset$. Let $y^{\prime} \in h\left(G_{1}\right) \cap h\left(G_{2}\right) \cap J_{Y}$. Since $G_{1} \cap G_{2}=\emptyset, h^{-1}\left(y^{\prime}\right)$ 
is not a singleton, implying that $h \mid J_{X}: J_{X} \rightarrow J_{Y}$ is not one-to-one. This contradicts the conclusion of step 1.

Finally, since $h\left(J_{X}\right) \cap h\left(X-J_{X}\right)=\emptyset$, it follows from steps 1 and 2 above that $h$ is one-to-one.

Proof of Theorem 1.2: By Theorem 2.1, if $Y$ is not homeomorphic to $[0,1]$ or the one-point space, then $Y$ is a compactification of the ray with the arc as remainder. By Lemma 2.2, $h$ is one-to-one. Since $X$ is compact and $Y$ is Hausdorff, it follows that $h$ is a homeomorphism.

Finally, the following example shows that Theorem 1.2 does not hold for confluent or even monotone maps.

EXAMPLE. Let $X$ and $Y$ be the continua shown below

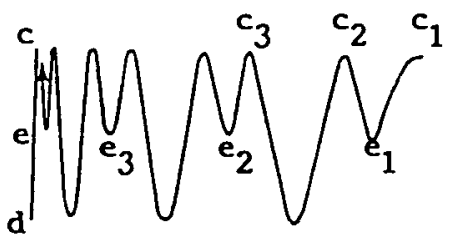

$\mathbf{X}$

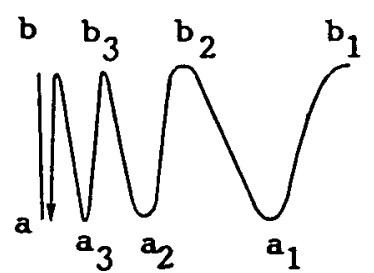

$\mathbf{Y}$

For each $i \in N$, let $K_{i}=\left\{t \in\left[c_{2 i}, c_{2 i+1}\right]: \pi_{2}(t) \leqslant e\right\}$. Define $h: X \rightarrow Y$ as follows:

$$
\begin{aligned}
& \text { (i) } h\left(c_{i}\right)=b_{i} \\
& \text { (ii) } h\left(e_{i}\right)=a_{2 i-1} \\
& \text { (iii) } h\left(K_{i}\right)=a_{2 i} \\
& \text { (iv) } h[e, d]=a \text { and } h(c)=b \\
& \text { (v) } h \text { is extended linearly on the rest of } X .
\end{aligned}
$$

It is easy to check that $h$ is a monotone (hence confluent) map, and that $X$ is not homeomorphic to $Y$.

Other examples of monotone and confluent onto maps between nonhomeomorphic and fairly complicated compactifications of the ray with the arc as remainder may be constructed. This suggests that characterising confluent images of an arbitrary compactification is more involved than the one obtained in [6] for the $\sin (1 / x)$ continuum.

\section{REFERENCES}

[1] Marwan M. Awartani, 'Compactifications of the ray with the arc as remainder', Topology Proc. 9 (1984), 201-215. 
[2] Marwan M. Awartani, 'An uncountable collection of mutually incomparable chainable continus' (to appear).

[3] J.J. Charatonik, 'Confluent mappings and unicoherence of continua', Fund. Math. 56 (1964), 213-220.

[4] J.L. Kelley, 'Hyperspaces of a continuum', Trans. Amer. Math. Soc. 52 (1942), $22-36$.

[5] A. Lelek, 'Some problems concerning curves', Colloq. Math. 23 (1971), 93-98.

[6] Sam B. Nadler Jr., 'Confluent images of the sinusoidal curve', Houston J. Math. 3 (1977), 515-519.

[7] Sam B. Nadler Jr., 'Multicoherence techniques applied to inverse limits', Trans. Amer. Math. Soc. 157 (1971), 227-234.

[8] Sam B. Nadler Jr., 'Arc components of certain chainable continua', Canad. Math. Bull. 14 (1971), 183-189.

[9] Roger W. Wardle, 'On a property of J.L. Kelley', Houston J. Math. 3 (1977), 291-299.

Department of Mathematics

Faculty of Science

United Arab Emirates University

P.O. Box 15551, Al-Ain

United Arab Emirates 